\title{
Oral Care Practices of Nurses on Ventilated Patients in Kigali Intensive Care Unit
}

\author{
Liberatha Rumagihwa ${ }^{1 *}$, Busisiwe Rosemary Bhengu ${ }^{2}$ \\ 1 School of Nursing and Midwifery, University of Rwanda, College of Medicine and Health Science, Kigali, Rwanda \\ ${ }^{2}$ New York University, Rory Meyers College of Nursing, New York, US A
}

*Corresponding author: Liberatha Rumagihwa. School of Nursing and Midwifery, College of Medicine and Health Sciences, University of Rwanda, Remera Campus, 11 KG 47, Kigali, Rwanda. Email: rumaglib@yahoo.fr

\begin{abstract}
Background

Oral care in the Intensive Care Unit (ICU) is an essential intervention proven to prevent Ventilator Associated Pneumonia (VAP). VAP results in increased morbidity and mortality, with excess hospital stay and cost. Oral care is a critical intervention that controls the accumulation of oral pathogens; however, it is viewed as a comfort measure and not a critical life-saving intervention.

Objective
\end{abstract}

To assess the oral care provided to ICU ventilated patients by nurses at a referral hospital in Kigali.

\section{Methods}

This cross-sectional design used a convenience sample of 47 nurses at a referral hospital in Kigali. Descriptive statistics were used for data analysis.

\section{Results}

The majority of nurses $(89.4 \%)$ used normal saline for comprehensive mouth care on ICU patients. The majority reported they never used a toothbrush $(53.2 \%)$ toothpaste $(63.8 \%)$, or Chlorhexidine solution (89.4\%), which are the recommendation. One third (34\%) do not perform the oral assessment on admission. There is no ICU oral care protocol, and oral hygiene supplies are limited.

\section{Conclusion}

Oral care is critical care in VAP prevention. ICU nurses reported a low level of oral care practice. We recommend an oral care protocol and in-service training to improve the quality of oral care to ICU ventilated patients.

Rwanda J Med Health Sci 2019;2(2):154-159.

Keywords: Oral care, ICU, nurses, ventilated patients, ventilator-associated pneumonia.

\section{Background}

Oral care is essential to ventilated patients in the intensive care unit (ICU) in order to help prevent nosocomial infections.[1] Ventilated-acquired pneumonia (VAP) is the most fatal nosocomial infection, [2] which affects $10 \%$ to $20 \%$ of ventilator patients, with almost twice the mortality rate than those without VAP.[1,2] VAP develops after 48 hours of intubation,[3] and causes increased morbidity and mortality, and excess hospitalization and costs. [1,2] Oral care in critically ill patients is primarily a safety measure, though also a comfort measure and a nursing responsibility, as ventilated patients are not able to perform oral self-care.[4] The practice of oral care in ICU is not consistent,[4] and nurses do not make oral care a priority.[5]

Nosocomial infections pose a significant safety concern for both patients and healthcare providers (HCPs).
[6] Ventilated patients are more at risk as intubation compromises the integrity of the oropharynx and trachea, and allows oral and gastric secretions to enter the normally sterile respiratory tract.[7] Bacteria can translocate to the lungs easily in compromised patients because the natural body defenses that maintain homeostasis of the oropharynx and respiratory system are impaired. $[7,8]$ The most common bacteria found in a five-year longitudinal study (2009-2013) of 5298 isolates in Rwanda included, Escherichia coli (46.7\%), Klebsiella spp. (18.4\%), Staphylococcus aureus (11.7\%), Enterococcus spp. (10.3\%), Psendomonas spp. (7.1\%), and Acinetobacter spp. (5.9\%). [9] Another study in Rwanda,[10] found the three most common multidrugresistant (MDR) pathogens included E. coli, Klebsiella, and S. aureus isolates, though the type of causative and drugresistant pathogen changes depend on locality.

Oral hygiene decreases the oropharynx bacteria and stimulates saliva flow, which contains many protective 
immunoglobulins to reduce plaque and pathogens. [7,9] A recent Cochrane systematic review with 6016 participants, compared four oral hygiene interventions such as chlorhexidine ( $\mathrm{CHX}$ ) solution or gel versus placebo or usual care; tooth brushing versus no tooth brushing; manual versus powered tooth brushing; and various oral care solutions.[3] The review concluded that CHX mouthwash or gel was the most effective solution.[3] The review revealed a VAP reduction of $6 \%$ (from $24 \%$ to $18 \%$ ) compared to usual care, or placebo in critically-ill patients (RR $0.75,95 \%$ CI 0.62 0.91, $\mathrm{p}=0.004, \mathrm{I} 2=35 \%$ ).[3] However, a retrospective study of 5500 ventilated patients revealed the daily use of CHX solution decreased VAP rates, but increased mortality (HR 1.63; 95\% CI 1.15-2.31; $\mathrm{p}=0.006$ ), that was likely related to aspiration and attendant respiratory distress syndrome. [8] However, the Cochrane Review [3] indicated there was no increased mortality rate.

Numerous studies have focused on oral care of ventilated patients by ICU nurses. A study in Sudan, indicated a high level of knowledge $(97.4 \%)$ related to the importance of oral care in ICU patients, though only $20 \%$ had good oral care practice.[11] The study emphasized a need for an ICU oral care protocol and training.[11] A similar study in Iran showed oral care was not a priority, due to staff shortages and writing duties, though only $20 \%$ of patients' charts contained nurses' notes about oral care.[5] In Dubai, a pre-test and post-test educational intervention among 6016 participants showed a significant increase in knowledge and oral hygiene practice.[12]

This study intended to assess the level of oral care practice of nurses caring for ventilated patients in the ICU at one referral hospital in Rwanda. There does not appear to be a publication on this specific topic. The results of the study might prompt nurses and physicians in the ICU referral hospital to develop an oral care protocol, and also inform other clinical hospital administrators to adopt the protocol. Results of the study might improve ICU patient care, and consequently ICU patient care outcomes throughout Rwanda. This study aimed to assess the level of knowledge and practice of comprehensive mouth care provided by nurses to ventilated patients in the ICU at a referral hospital in Kigali.

\section{Methods}

\section{Design}

This study was a cross-sectional design to assess the knowledge and practice of ICU nurses on the Adult Intensive Care Unit (AICU) and Pediatric Intensive Care Unit (PICU) of a referral hospital. The study was conducted at the University Teaching Hospital in Kigali (UTHK), which is the leading public health institution in the country and located in Rwanda's capital city. The study was conducted during March and April 2017.

\section{Participants' recruitment}

The participants were 47 registered nurses working in the ICU (adult and pediatric) at UTHK. The nurses had various levels of nursing education and work experiences.

\section{Measures}

The questionnaire for the current study was adapted from a part of a Master's dissertation at the University of Kwazulu Natal, in South Africa.[13] Approval was given to use and adapt the instrument. The questionnaire consisted of closed questions to answer the study objectives, and an additional area was available to add comments as needed. The questionnaire consisted of 45 questions and included the following:

Section A: Demographic characteristics, (6 questions) (age, gender, qualification category, length of nursing experience, length of ICU experience, and nationality) Section B: Knowledge of oral care practice (13 questions) (1 point for correct answer, 0 for wrong answer)

Section C: Perceptions of oral care (8 questions) (on the availability and adherence to oral care protocol). Scoring: Yes or No.

Section D: Oral care practices (18 questions). Scoring of the practice questions: [14]

- "Good" oral care practice scored $\geq 75 \%$

- "Moderate" practice scored 50-74\%

- "Poor" practice scored $\leq 49 \%$

For multiple choice questions with many options, 1 point was given for the correct answer, and 0 points for any other answer. A reliability test of the instrument resulted in a Cronbach's alpha of 0.74 for the practice questions.

\section{Data collection}

The study was explained to staff and then invited to participate, knowing that their responses would be confidential, anonymous, and able to withdrawal at any time. Details about the study were explained after the morning staff meeting, and study participants agreed to complete the questionnaires during their break time to avoid disruption of the ward routine activities. Nurses who agreed to participate signed the informed consent before data collection. The questionnaire took about 15 minutes to complete. The investigator was responsible for distributing and collecting the completed questionnaires.

\section{Data analysis}

Descriptive statistics were used to calculate frequency distributions and Chi-square for relationships among variables. A significance level of 0.05 was used. Data were analyzed with SPSS version 21. 


\section{Ethical considerations}

Approval for the research was obtained from the University of Rwanda, College of Medicine and Health Sciences Institutional Review Board, UTHK Ethics Board, and the ICU unit managers. Informed consent was obtained from all participants, and confidentiality and anonymity were maintained.

\section{Results}

Over three-quarters of participants $(78.7 \%)$ surveyed had self-reported a "poor" level of practice, while nearly a quarter $(21.3 \%)$ self-reported a "moderate" level of practice (figure 1). None rated themselves at a "good" level of oral care practice.

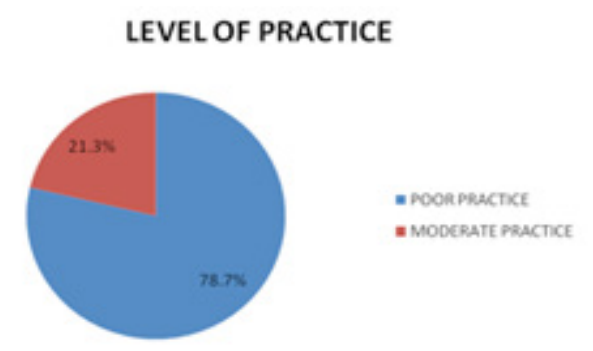

Figure 1. ICU nurses' self-reported level of practice for ventilated patients

One-third of participants (34\%) reported that they do not examine the patient's mouth on admission to the ICU, though two thirds $(66.0 \%)$ perform comprehensive mouth care daily. (table 1$)$.

Table 1. Assessment of oral health

\begin{tabular}{ll}
\hline $\begin{array}{l}\text { Frequency of oral } \\
\text { Assessment }\end{array}$ & $\mathbf{n ~ ( \% )}$ \\
\hline On ICU admission & \\
No & \\
Yes & $16(34.0)$ \\
Daily assessment in ICU & $31(66.0)$ \\
Not at all & $1(2.1)$ \\
Once a day & $31(66.0)$ \\
Twice a day & $3(6.4)$ \\
Three times a day & $2(4.3)$ \\
$\geq 4$ times a day & $1(2.1)$ \\
As needed & $9(19.1)$
\end{tabular}

The majority who provided oral care to the ICU ventilated patients reported that they have never used a toothbrush $(53.2 \%)$ or toothpaste $(63.8 \%)$ while performing comprehensive mouth care on patients (table 2). The majority of particpants reported washing the patient's mouth out with normal saline (89.4\%), whereas a few used tap water $(14.9 \%)$, or Chlorhexidine solution $(10.6 \%)$.
Table 2. Oral Care Interventions of ICU Ventilated Patients

\begin{tabular}{llll}
\hline Intervention & $\begin{array}{l}\text { Never } \\
\mathbf{n}(\mathbf{\%})\end{array}$ & $\begin{array}{l}\text { Rarely } \\
\mathbf{n}(\mathbf{\%})\end{array}$ & $\begin{array}{l}\text { Always } \\
\mathbf{n}(\mathbf{\%})\end{array}$ \\
\hline Toothbrush & $25(53.2)$ & $12(25.5)$ & $10(21.3)$ \\
Toothpaste & $30(63.8)$ & $14(29.8)$ & $3(6.4)$ \\
Swab & $4(8.5)$ & $7(14.9)$ & $36(76.6)$ \\
Sterile water & $19(40.4)$ & $10(21.3)$ & $18(38.3)$ \\
Tap water & $7(14.9)$ & $7(14.9)$ & $33(70.2)$ \\
Normal saline & $0(0)$ & $5(10.6)$ & $42(89.4)$ \\
Chlorhexidine & $42(89.4)$ & $5(10.6)$ & $0(0)$ \\
& & & \\
\hline
\end{tabular}

Three-quarters of participants $(74.5 \%)$ responded that there was no protocol on comprehensive oral care in the ICU where they worked (figure 2).

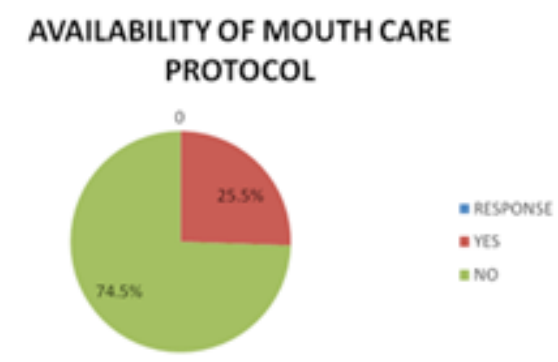

Figure 2. ICU Oral Care Protocol

The majority of participants $(85.1 \%)$ were never trained to perform comprehensive mouth care on ventilated patients in their basic nursing training, whereas only a small proportion $(14.9 \%)$ responded they had been trained (table 3). Furthermore, the majority (80.9\%) revealed they had not received training on oral assessment or oral care on ventilated patients since appointed to work in the ICU. A small proportion $(19.1 \%)$ had received training on mouth care that was identified as in-service training (12.8\%). 
Table 3. Oral Care Training of ICU Nurses

\begin{tabular}{ll}
\hline Training & n (\%) \\
\hline $\begin{array}{l}\text { Did you receive assessment } \\
\text { and provision of comprehensive } \\
\text { oral care in basic nursing training? }\end{array}$ & \\
No & $40(85.1)$ \\
Yes & $7(14.9)$ \\
Did you receive oral care training? & \\
No & $27(57.4)$ \\
Yes & $20(42.6)$ \\
Have you received training in & \\
assessment and provision of & \\
oral health care in ICU? & \\
No & \\
Yes & $38(80.9)$ \\
If yes, what kind of training? & $9(19.1)$ \\
No training & $41(87.2)$ \\
In service & $6(12.8)$ \\
Would you like further training & \\
on assessment and provision \\
of comprehensive oral care?
\end{tabular}

\section{Discussion}

The findings of the study suggest that there is a low level of knowledge and for practice of oral care among the study population at the referral hospital.

\section{Knowledge of comprehensive oral care}

More than half $(57.4 \%)$ of participants knew various procedures that might be considered comprehensive mouth care, but few knew that poor oral care might result in VAP. Similarly, a study in Iran indicated only $21 \%$ of ICU nurses understood the reason for oral care was to prevent VAP.[15] In contrast, a study in Khartoum State, Sudan revealed that almost all nurses $(97.4 \%)$ stated that the provision of oral care in ICU is essential for infection prevention.[11] A third (34\%) of ICU nurses showed that they did not assess patients on admission, while two-thirds did (66.0\%), similar to the study in Iran that showed that more than one-fifth did not do an oral assessment.[15]

\section{Perception of comprehensive oral care}

Three quarters $(76 \%)$ of the participants perceived comprehensive mouth care at a high level, and over a quarter rated it as an essential procedure for ventilated patients $(36.2 \%)$; this is in contrast with an Iranian study which showed that on a scale of 1-10 nurses rated oral care with a score of 5.7.[15] There was a significant correlation $(p<0.000)$ between knowledge and perception, which was similar to a study in Khartoum State, Sudan $(\mathrm{p}<0.000) \cdot[11]$

\section{Practices of comprehensive oral care}

The majority $(78.7 \%$ ) of participants had a low level of oral care practice on ventilated patients, which was likely linked to a lack of protocol in the ICU. In Iran only a quarter $(21.5 \%)$ of nurses were not providing oral care to patients, indicating that over three-quarters of nurses were providing oral care.[15] Various studies have shown that a standardized ICU protocol would improve the quality of oral care provided to ventilated patients. $[16,17]$ Over half of those surveyed $(53.2 \%)$, reported that they had never used a toothbrush while performing comprehensive mouth care, whereas numerous studies indicated a toothbrush was the best tool if used twice a day to remove plaque. $[16,18,19]$

Our findings showed that the majority (89.4\%) did not use the CHX solution, yet high-quality evidence from 18 RCTs showed that CHX mouth rinse or gel reduced the risk of VAP from $24 \%$ to18\%, compared to placebo or usual care.[3] CHX was also proven to be an essential mouthwash among patients hospitalized in ICU through numerous studies.[17,20,21] Normal saline was used by the majority (89\%) of participants working in the ICU. However, a study revealed normal saline has a drying effect on the oral mucosa and not recommended;[16] and therefore would exacerbate an already dry mouth in a ventilated patient.

Nearly half $(48.9 \%)$ of participants mentioned there is no provision of oral hygiene hospital supplies. A study in India showed similar findings, with nearly three quarters $(72 \%)$ of nurses complaining of inadequate supplies and equipment.[15] The participants gave conflicting responses, such as over half $(59.6 \%)$ said that they did not have any difficulty performing comprehensive oral care on ventilated patients, and yet the results showed that over three quarters $(78.7 \%$ ) had a low level of practice. Indeed, if supplies are not available, that would hinder regular oral patient care.

\section{In-service training}

The majority $(85.1 \%)$ had never learned how to perform comprehensive oral care in ventilated patients in their basic nursing training; therefore, very few $(14.9 \%)$ were trained. Furthermore, the majority $(80.9 \%)$ stated that they had not received in-service oral hygiene training in the ICU, though a limited proportion of ICU nurses $(19.1 \%)$ had received training.

However, nearly all $(91.5 \%)$ would appreciate oral assessment and provision of comprehensive mouth care in the future. A few $(8.5 \%)$ declined further training believing they were competent in oral care. This situation is somewhat problematic as oral care on ventilated patients requires specific knowledge and skills compared to patients in general ward. Similarly, two other studies indicated over half of the nurses did not pass any post basic oral care training. [11,15] In contrast, 
a study in Sudan revealed that most nurses had received basic training in oral care.[11] The results of the current study are alarming, as researchers have indicated in service training provides new employees with extensive basics of intensive care and work with patients with life threatening conditions independently.[22]

\section{Educational programs}

Continuous educational sessions are essential for ICU nurses in maintaining good oral care practices with ventilated patients.[3] An oral care educational session in South Africa resulted in significant improvement of practices and clinical outcomes.[23] Similarly, an educational program in Egypt indicated a low level of knowledge in a pre-test, that increased significantly in the post-test after an educational intervention.[24] Furthermore, the study not only improved the nurses' oral care knowledge and skills but also increased their motivation to practice oral care. [24]

In contrast, a study in Finland found minimal effects in a single-dose simulation education session, even though it had a structured debriefing and verbal feedback on critical care nurses' knowledge and skills.[18] Comprehensive oral care programs that improve efficiency and efficacy should be carefully developed, implemented and regularly evaluated in order to ensure consistency and high quality of standardized care. [25] The survey conducted in India showed that additional education is necessary to increase awareness of the relationship between dental plaque and the patient's systemic conditions.[26]

\section{Oral care protocol}

In the present study, more than three quarters $(76.6 \%)$ of participants reported that there was no mouth care protocol in ICU to guide oral care practice, and this was likely linked to the poor oral care performance. A comprehensive oral care protocol and attendant compliance can significantly reduce VAP rates. [21,27] Similarly, a study in Sudan showed not having a protocol influenced nurses' practice.[11] Furthermore, the presence of service delivery protocol rendered bedside dentistry services delivery more organized on the unit.[28]

\section{Limitations}

The sample size was small, and the study was conducted at only one hospital, so the results cannot be generalized to other hospitals or regions.

\section{Future research}

A larger sample comparing other ICU facilities would be the next step. New nursing graduates from the University of Rwanda MScN Critical Care track are also in a prime position to become change agents and initiate and help sustain the development of a patient-care oral protocol at the site where this study was conducted.

\section{Conclusion}

This study assessed the level of knowledge and practice of comprehensive mouth care provided to ICU ventilated patients by nurses at a referral hospital in Kigali. The findings showed that the majority of ICU nurses had a low level of oral care practice among patients in the ICU, which is a concern. Evidence has shown that comprehensive mouth care is a preventive measure of VAP, in addition to being a comfort measure. This situation was highly linked to the lack of in-service training and the absence of a protocol. The present study highlighted the need for establishing an oral hygiene protocol for ventilated patients and providing regular in-service training.

\section{Acknowledgments}

I thank the University of Rwanda administration and academic staff for their support very much, and I am also thankful to Rudo Nyamakura who guided and advised me with parental words and supporting ideas, many thanks to Dr. Pamela Meharry for her endless emotional and physical support with this manuscript.

This article is published open access under the Creative Commons Attribution-NonCommercial NoDerivatives (CC BY-NC-ND 4.0). People can copy and redistribute the article only for noncommercial purposes and as long as they give appropriate credit to the authors. They cannot distribute any modified material obtained by remixing, transforming or building upon this article. See https:/ / creativecommons.org/licenses/by-nc-nd/4.0/

\section{References}

1. Torres A, Niederman MS, Chastre J, Ewig S, Fernandez-vandellos P, Hanberger H, et al. Summary of the international clinical guidelines for the management of pneumonia. 2018; Available from: http://dx.doi.org/10.1183/23120541.00028-2018

2. Vallés J, Pobo A, Real J. Excess ICU mortality attributable to ventilator-associated pneumonia: The role of early vs late onset. 2007;1363-8.

3. Hua F, Xie H, Hv W, Furness S, Zhang Q, Li C, et al. Oral hygiene care for critically ill patients to prevent ventilator-associated pneumonia ( Review ) Oral hygiene care for critically ill patients to prevent ventilator-associated pneumonia. 2017;

4. Chacko R, Rajan A, Lionel P, Thilagavathi M, Yadav B, Premkumar J. Oral decontamination techniques and ventilator-associated pneumonia. 2017;26.

5. Javadinia SA, Kuchi Z, Saadatju A, Tabasi M, Adib-Hajbaghery M. Oral care in trauma patients admitted to the ICU: Viewpoints of ICU nurses. Trauma Mon. 2014;19:7-12.

6. Mehta Y, Gupta A, Ramasubban S. Guidelines for prevention of hospital acquired infections. Indian J Crit care Med. 2014;18:149-63. 
7. Torres A, Niederman M, Chastre J, Ewig S, Fernandez-vandellos P, Fernandez-vandellos P, et al. International ERS / ESICM / ESCMID / ALAT guidelines for the management of hospitalacquired pneumonia and ventilator-associated pneumonia. 2017; Available from: http://dx.doi. org/10.1183/13993003.00582-2017

8. Klompas M, Branson R, Eichenwald EC, Greene LR, Howell MD, Lee G, et al. Strategies to Prevent Ventilator-Associated Pneumonia in Acute Care Hospitals: 2014 Update. 2014;35:915-36.

9. Booker S, Murff S, Lisa K, Jablonski R. Mouth Care to Reduce Pneumonia. 2013;113.

10. Carroll M, Rangaiahagari A, Musabeyezu E, Singer D. Five-Year Antimicrobial Susceptibility Trends Among Bacterial Isolates from a Tertiary Health-Care Facility in Kigali, Rwanda. 2016;

11. Ibrahim SM, Mudawi AM, Omer O. Nurses' Knowledge, Attitude and Practice of Oral Care for Intensive Care Unit Patients. 2015;179-86.

12. Cherian S, Karkada S. Effect of education related to oral care practices on nurses' knowledge, practice and clinical outcomes of mechanically ventilated patients in Dubai. Cherian, Sonia Karkada,Suja. 2015;2:9-14.

13. Sarepho AP. A dissertation submitted to The Faculty of Health Sciences, School of Nursing, University of Kwazulu Natal, Durban, South Africa Partial fulfilment of the requirements For the masters degree in critical care and trauma. 2011;

14. Elbokhary R, Osama A, Al-khader M. Knowledge and Practice of ICU Nurses Regarding Endotracheal Suctioning for Mechanically Ventilated Patients in Khartoum Teaching Hospital. Am J Clin Neurol Neurosurg. 2015;1:928.

15. Hajbaghery MA, Ansari A, Fini IA. Intensive care nurses' opinions and practice for oral care of mechanically ventilated patients. Indian J Crit Care Med. 2013;23-7.

16. Nobahar M, Reza M, Malek F, Ghorbani R. Original article Effects of hydrogen peroxide mouthwash on preventing ventilator-associated pneumonia in patients admitted to the intensive care unit. Brazilian J Infect Dis [Internet]. Sociedade Brasileira de Infectologia; 2016;20:44450. Available from: http://dx.doi.org/10.1016/j. bjid.2016.06.005

17. Hillier B, Wilson C, Chamberlain D, King L. Preventing ventilator-associated pneumonia through oral care, product selection, and application method: a literature review. AACN Adv Crit Care [Internet]. 2013;24:38-58. Available from: http://www.ncbi.nlm.nih.gov/ pubmed/23343813

18. Illsley A. Mouth care within Adult Critical care Anne Illsley, Service Improvement PDM, Ruth Pettit Deputy Sister, Critical Care Review of Guideline. Mouth care within Adult Critical care Anne Illsley, Service Improvement PDM , Ruth Pettit Deputy Sister, Criti. 2015;

19. Nicolosi N. L, Rubio C, Martinez CD, Edith M, Gonzalez N. Effect of Oral Hygiene and 0 . $12 \%$ Chlorhexidine Gluconate Oral Rinse in Preventing Ventilator-Associated Pneumonia After Cardiovascular Surgery. 2014;504-9.

20. Cuccio L, Cerullo E, Rivet C, Steeves S. An Evidence-Based Oral Care Protocol to Decrease Pneumonia. 2012;

21. Dale CM, Angus JE, Sinuff T, Rose L. Ethnographic investigation of oral care in the intensive care unit. Am J Crit Care. 2016;25:249-56.

22. Mateja S, Alma K. International Journal of Nursing \& Clinical Practices Education and Training for Quality Work at the Intensive Care Unit. Int J Nurs Clin Pract. 2016;3:3-6.

23. Snyders O, Khondowe O, Bell J. Oral chlorhexidine in the prevention of ventilator- associated pneumonia in critically ill adults in the ICU: A systematic review. South Africa J Crit Care. 2011;27:48-55.

24. Adly RM, Amin FM, Aziz MAA El. Improving Nurses' Compliance with Standard Precautions of Infection Control in Pediatric Critical Care Units. World J Nurs Sci. 2014;1-9.

25. Jansson MM, Syrjälä HP, Ohtonen PP, Meriläinen MH, Kyngäs HA, Ala-kokko TI. Effects of simulation education on oral care practices - a randomized controlled trial. 2017;22:161-8.

26. Miranda A, de Paula R, de Castro Piau CB, Costa P, Bezerra AB. Oral care practices for patients in Intensive Care Units: A pilot survey. Indian J Crit Care Med [nternet]. 2016;20:267. Available from: http://www.ijccm.org/text. asp?2016/20/5/267/182203

27. Garcia R, Jendresky L, Colbert L, Bailey A, Zaman M, Majumder M. Reducing ventilator associated pneumonia through advanced oral dental care: A 48 month study. 2009;18:523-34.

28. Blum DFC, Silva JAS da, Baeder FM, Bona Á Della. The practice of dentistry in intensive care units in Brazil. 2018;30:327-32. 